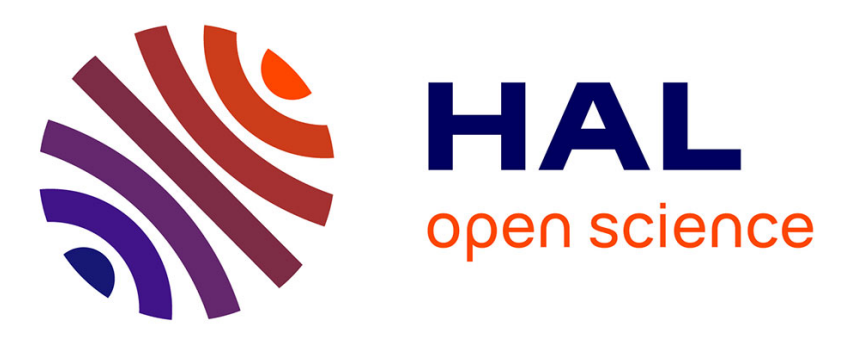

\title{
Quantitative Temperature Measurements in Gold Nanorods Using Digital Holography
}

\author{
Adrien Lalisse, Abeer Al Mohtar, Minh Chau Nguyen, Rémi Carminati,
} Jérôme Plain, Gilles Tessier

\section{To cite this version:}

Adrien Lalisse, Abeer Al Mohtar, Minh Chau Nguyen, Rémi Carminati, Jérôme Plain, et al.. Quantitative Temperature Measurements in Gold Nanorods Using Digital Holography. ACS Applied Materials \& Interfaces, 2021, 13 (8), pp.10313-10320. 10.1021/acsami.0c22420 . hal-03148575

\section{HAL Id: hal-03148575 \\ https://hal.sorbonne-universite.fr/hal-03148575}

Submitted on 22 Feb 2021

HAL is a multi-disciplinary open access archive for the deposit and dissemination of scientific research documents, whether they are published or not. The documents may come from teaching and research institutions in France or abroad, or from public or private research centers.
L'archive ouverte pluridisciplinaire HAL, est destinée au dépôt et à la diffusion de documents scientifiques de niveau recherche, publiés ou non, émanant des établissements d'enseignement et de recherche français ou étrangers, des laboratoires publics ou privés. 


\title{
Quantitative Temperature Measurements in Gold Nanorods Using Digital Holography
}

\author{
Adrien Lalisse ${ }^{1,2, \dagger}$, Abeer Al Mohtar ${ }^{1,3, \dagger}$, Minh Chau Nguyen ${ }^{4}$, Rémi Carminati ${ }^{3}$, Jérôme Plain ${ }^{2}$, Gilles \\ Tessier ${ }^{1,4, *}$ \\ ${ }^{1}$ Laboratoire de Neurophotonique CNRS UMR8250, Université Paris Descartes, 75270 Paris, France \\ ${ }^{2}$ Light, nanomaterials and nanotechnology L2n, UTT and CNRS ERL 7004, 12 rue Marie Curie - CS 42060, 10004 Troyes, \\ France \\ ${ }^{3}$ ESPCI Paris, PSL University, CNRS, Institut Langevin, 1 rue Jussieu, 75005 Paris, France \\ ${ }^{4}$ Sorbonne Université, INSERM, CNRS, Institut de la Vision, 17 rue Moreau, F-75012 Paris, France \\ *Corresponding author: gilles.tessier@sorbonne-universite.fr \\ ${ }^{+}$These authors contributed equally to this work
}

\begin{abstract}
Temperature characterization and quantification at the nanoscale remain core challenges in applications based on photoinduced heating of nanoparticles. Here, we propose a new approach to obtain quantitative temperature measurements on individual nanoparticles by combining modulated photothermal stimulation and heterodyne digital holography. From full-field reconstructed holograms, temperature is determined with a precision of $0.3 \mathrm{~K}$ via a simple approach without requiring any calibration or fitting parameters. As an application, the dependence of temperature on the aspect ratio of gold nanoparticles is investigated. A good agreement with numerical simulation is observed.
\end{abstract}

KEYWORDS: thermoplasmonics, digital holography, photothermal holography, gold nanostructures, optical modeling, thermal modeling.

\section{INTRODUCTION}

The excitation of electronic oscillations, plasmons, in non-perfect ohmic metals is necessarily associated to heat generation through Joule effect. Metal nanostructures can therefore be used to generate strong optical near-field enhancements, but also local heat sources. Historically, sub-diffraction optical confinement kick-started research in plasmonics. More recently, the possibility to create ultra-local heat sources has also found a lot of innovative applications, namely in photothermal cancer therapy ${ }^{1-3}$ nanosurgery, ${ }^{4}$ drug delivery, ${ }^{5-7}$ photothermal imaging, ${ }^{8}$ photoacoustic imaging, ${ }^{9}$ magnetic recording, ${ }^{10}$ nanochemistry, ${ }^{11}$ thermonics, ${ }^{12}$ optofluidics, ${ }^{13}$ and temperature shaping. ${ }^{14}$ However, despite the recent progress of the thermoplasmonics field, quantitative temperature measurements at the nanoscale remain a real challenge. To access this local information, scanning probes and far-field optical techniques have been developed. The near-field techniques, initiated in 1986 by Williams and Wickramasinghe, ${ }^{15}$ rest upon the use of a sharp AFM-type tip associated to a temperature sensor. Although their spatial resolution is unrivalled, nearfield techniques are inherently invasive and difficult to interpret quantitatively due to the complexity of tip-sample thermal interactions.

Far-field optical techniques can circumvent these limitations. While infrared-based methods offer limited spatial resolution, fluorescence-based methods are widespread today: fluorescence polarization anisotropy ${ }^{16}$, fluorescence intensity ${ }^{17,18}$, luminescent molecular thermometers ${ }^{19}$ or fluorescence spectroscopy ${ }^{20,21}$ can provide fast, accurate and spatially resolved temperature measurements. However, these measurements can suffer from photo- or thermo-bleaching and can be sensitive to the local fluorophore environment ( $\mathrm{pH}$, ions and viscosity), their main drawback is that they are intrinsically invasive: fluorophores must be incorporated to the sample, which is straightforward in biological applications, but much less so in plasmonics.

Recently, Raman spectroscopy has emerged as a major tool for quantitative temperature measurements in silicon ${ }^{22}$, or gold ${ }^{23}$ that was first demonstrated by Hugall et al. ${ }^{24}$ for distributed structures and Xie et al. ${ }^{25}$ for individual nanostructures. Techniques to enhance the signal, thus reduce the read-out time, have been proposed through the introduction of plasmonic ${ }^{26}$ or nonplasmonic nanoparticles $^{22}$, or a combination of both ${ }^{27}$. However, Raman spectroscopy still requires careful calibrations and relatively long integration times to measure the temperature of a single point. While peerless as nanoscale-thermometers, these techniques are not adapted to imaging. 
Methods relying on the temperature dependence of the refractive index $n$ with temperature $T$ are entirely non invasive, fast, and sensitive enough to study single objects ${ }^{28}$. In some cases, they have the advantage of being able to probe the temperature of the particle rather than that of its environment ${ }^{29}$, and can be used to localize single nanoparticles or molecules ${ }^{8,30}$. However, using them to deduce the temperature quantitatively remains a challenge. $\mathrm{n}(\mathrm{T})$ can be considered linear over a few tens of Kelvins for most metals or dielectrics ${ }^{31}$, for higher temperature an iterative numerical approach can be followed, as proposed by Baffou et al. ${ }^{29}$. In simple structures, e.g. bulk materials or flat thin films, measuring the refractive index, usually through related properties such as reflectivity ${ }^{32,33}$ or optical path difference ${ }^{29}$ is therefore a straightforward way to measure temperature.

In the present work, we propose the use of heterodyne digital holography to image thermally-induced scattering modulations. Combined with a simple analysis, it provides fast (tens of seconds), sensitive $(0.3 \mathrm{~K})$ calibration-free (no adjustable parameter), noninvasive (no contrast agent) full field imaging ( $1024^{2}$ pixels) of high-frequency (up to the $\mathrm{MHz}$ ) modulated temperature components on individual nanostructures. A specially designed and fabricated array of nanoellipsoids with varying aspect ratios allowed us to validate the method by comparison with numerical simulations, based either on a finite difference time domain electromagnetic method coupled to thermal simulations (Lumerical), or on the boundary element method. This extensive analysis validates heterodyne digital holography as a reliable single nano-object thermal imaging technique.

\section{EXPERIMENTAL MATERIALS}

\subsection{Sample design and fabrication}

Plasmonic nanostructures offer an elegant way to control absorption and temperature at small scales. Whenever local optical enhancement is the main target, in e.g. plasmonic detection, heating is usually detrimental to the preservation of the plasmonic structure and/or the analyte, and should be minimized. Conversely, most thermoplasmonic applications demand efficient heat generation. Recently, we have proposed ${ }^{34}$ criteria to assess the ability of a given plasmonic system to enhance local or optical thermal phenomena. Resonances can indeed be tuned by changing the size, morphology, and composition of metallic nanostructures. A given volume of a chosen metal can therefore be shaped in order to maximize or minimize heat generation, depending on the application.

In this study, we chose a simple visible-resonating plasmonic system as reference: a flat gold cylinder, with a radius of $50 \mathrm{~nm}$ and a thickness of $20 \mathrm{~nm}$, i.e. a volume of $1.6 \times 10^{5} \mathrm{~nm}^{3}$, or approximately $9.2 \times 10^{6}$ gold atoms. The shape of the disc was then modified in order to investigate the temperature reached by various single structures of similar volume. Exploring all possible forms is of course impossible, and we restricted the present study to a class of shapes which can be fabricated relatively easily: cylinders with elliptic bases. As illustrated in Figure 1 (a) this family of objects is described by its aspect ratio $\mathrm{AR}=a / b$ where $a$ is the long axis and $b$ the short axis of the ellipse. We fabricated several identical series of objects ranging from discs (AR=1) to rod-like elongated ellipses $(\mathrm{AR}=9)$ in steps of $\triangle \mathrm{AR}=0.5$.

The nanostructures were fabricated on a $1 \mathrm{~mm}$ thick float glass substrate using e-beam lithography (EBL). The substrate was sonicated several times in deionized water and detergent for 15 minutes. Remaining particles were removed from the surface with deionized water (5 minutes), acetone (5 minutes), ethanol (10 minutes) and isopropanol (IPA) (15 minutes). A

polymethylmethacrylate (PMMA) electron-sensitive resist was then spin-coated on the cleaned glass substrate and annealed for 15 minutes at $170{ }^{\circ} \mathrm{C}$. A conductive resist layer (Espacer 300Z) was also spin-coated on top of the PMMA to prevent charging effects during the EBL exposure. The areas of interest were then exposed in an EBL machine (RAITH e-line) under a $15 \mathrm{kV}$ voltage. After exposure, the conductive resist layer was removed in deionized water, and the exposed PMMA was revealed in a cold mixture of methyl isobutyl ketone (MIBK) and isopropanol (IPA). An evaporator was then used to deposit 3 nm of titanium dioxide as an adhesive layer, and $20 \mathrm{~nm}$ gold. To dissolve the remaining PMMA and lift-off metal from the unexposed regions, the sample was put in acetone for 4 hours. Finally, the sample was rinsed with IPA and exposed to an $\mathrm{O}_{2}$ plasma before Scanning Electron Microscopy (SEM), and holographic characterizations. As can be seen in Figure 1 (b), the fabricated objects are close to the targeted geometry. However, SEM revealed that the narrowest and most elongated structures $(A R>5)$ were too thin to allow ideal fabrication, and exhibited either discontinuities (these were discarded) or were slightly wider than expected ( $b$ above the targeted value) due to the limited resolution of the EBL system. Dark-field optical spectroscopy on individual objects ${ }^{35}$ revealed a good agreement $( \pm 10$ $\mathrm{nm}$ on the resonance wavelengths) between the calculated and measured spectra, confirming a good precision in the fabrication, particularly regarding the length $a$ of the structures. Scanning Electron Microscopy carried out on selected structures (see Figure 1) indicated a fabrication precision of $\pm 5 \mathrm{~nm}$ on the length $a$ of the ellipses.

\subsection{Spectroscopic characterization}

Nanostructures were illuminated using unpolarized tungsten-halogen light and an air Dark-Field (DF) condenser, while the scattered light was collected by a lower numerical aperture microscope objective $(60 \mathrm{X}, \mathrm{NA}=0.7)$, and sent either to a fast camera (Andor Zyla CMOS) for direct DF imaging, or towards a line-imaging spectrometer (400-800 nm, Inspector V8E Specim) coupled to a second camera (Andor Ixon CCD). The measurement arm was equipped with an analyzer. After calibration with a Hg lamp, a reference spectrum $\mathrm{I}_{\text {ref }}$ accounting for source, transmission and detection efficiencies was acquired. Relevant nano-objects were identified with the imaging camera and positioned at the same location to ensure identical illumination conditions. Scattering $\mathrm{I}_{\text {scat }}$ and background noise $\mathrm{I}_{\text {bgd }}$ were measured simultaneously, at the nanostructure position and in a neighboring dark region, to derive normalized spectra $\mathrm{I}(\lambda)=\left(\mathrm{I}_{\text {scat }}-\mathrm{I}_{\mathrm{bgd}}\right) / \mathrm{I}_{\text {ref }}$ for each individual nano-object ${ }^{36}$.

Scattering spectra were modelled using MNPBEM $^{37}$ for a polarization along the short axis of the structures. Here, plasmonic resonances along the long axis were not modelled since they are essentially in the infrared, beyond the sensitivity range of our system. 
Figure 1 (c) shows the simulated spectra for aspect ratios AR varying from 1 to 4 which exhibit a Lorentzian shape characteristic of plasmon resonance, and a blue shift of the resonance at larger AR, as the short axis dimension decreases

With the exception of the AR = 1.5 structure, the simulated and experimental spectra (Figure 1. (c) and (d) respectively) follow a similar blueshift and decrease of the scattering with AR. Experimental spectra, however, are significantly broader, which is consistent with the roughness and irregularities observed by SEM (Figure 1 (b), typically of the order of $5 \%$. As illustrated by the AR=1.5 structure in the spectra of Figure 1 (d), some nanoobjects inevitably deviate from the intended shape. A systematic SEM control of the nanoparticles being impractical, we chose to mitigate such deviations by averaging multiple objects of identical intended geometry. As discussed below, these morphological variations, although limited, are the main source of variations in the observed temperatures, and far outweigh the measurement noise.
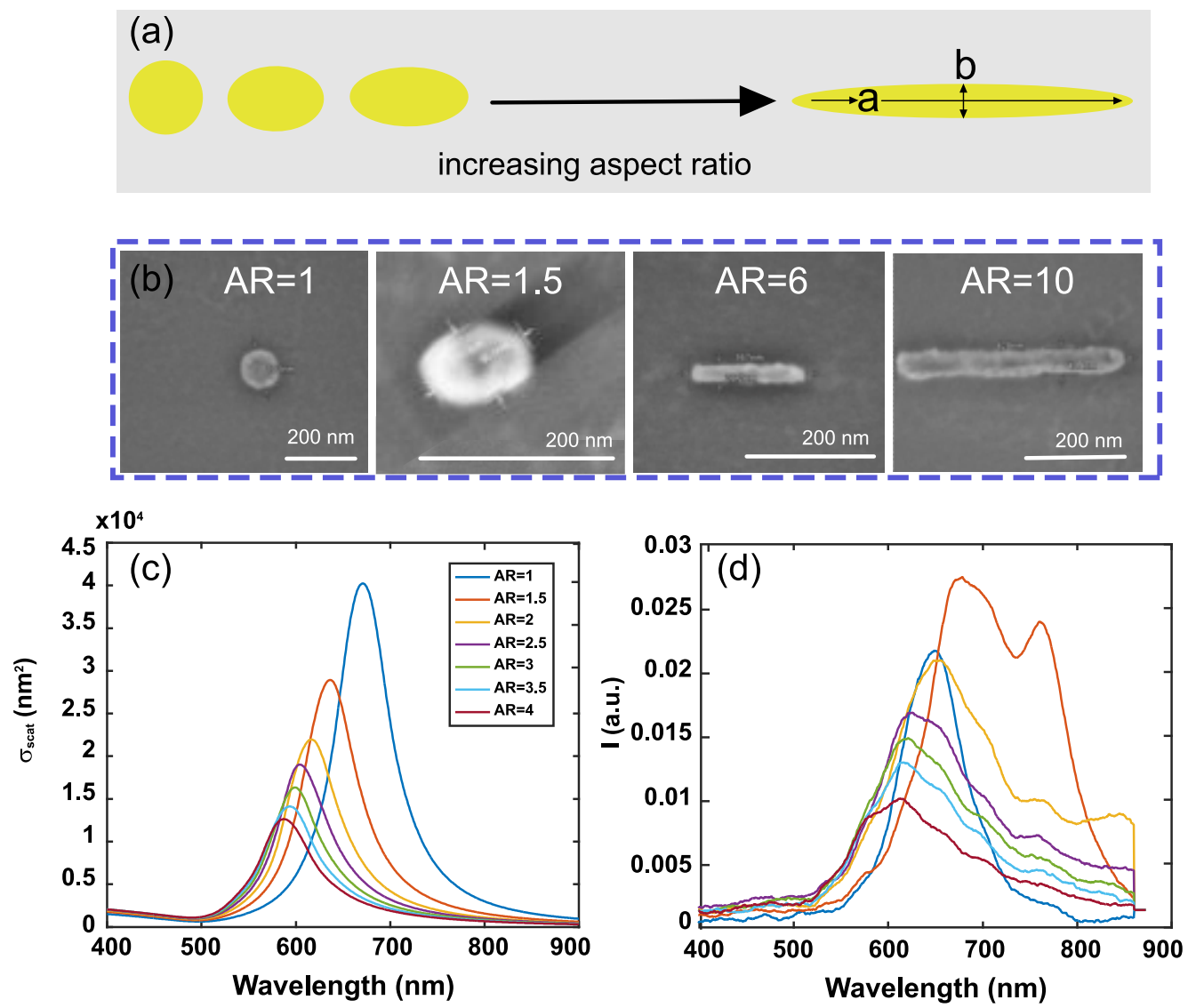

Figure 1. (a) Schematic of the sample geometry. (b) Typical SEM images of four nanorods of different aspect ratios (AR). The radius of the $\mathrm{AR}=1$ structure is $50 \mathrm{~nm}$. (c) Scattering spectra simulated using a Boundary Elements Method for AR 1 to 4, for a polarization perpendicular to the long axis of the structures, and (d) scattering spectra measured for a polarization perpendicular to the long axis of the structures on individual structures using white, dark field illumination. N.B.: spectra and SEM images were not measured on the same samples.

\section{EXPERIMENTAL METHODS}

\subsection{Description of physical mechanisms}

In order to probe temperature, we have chosen to rely on far-field measurements of the light scattered by the nanostructures. The modulated optical power $(\Delta P)$ sent to the sample induces a local temperature modulation $(\Delta T)$, which changes the refractive index of the object and its surroundings $(\Delta n)$, and in turn modulates the optical scattering $\left(\Delta I_{\text {scat }}\right)$, a process which can be summarized as:

$$
\Delta P \rightarrow \Delta T \rightarrow \Delta n \rightarrow \Delta I_{\text {scat }}
$$

However, thermo-optical coefficients are typically on the order of $10^{-4}$ to $10^{-5} \mathrm{~K}^{-1}$, and optical scattering by single nano-objects is very weak, which leaves us with the challenge of measuring small changes in a small signal. This can only be achieved in a modulated regime, taking advantage of lock-in type techniques. In order to avoid high order effects, we have chosen to dissociate the thermal excitation and the probe laser: The structures are heated using a modulated, high power laser, and a low power continuous probe laser is then used to measure the resulting variations in the scattered field. The modulated excitation induces both continuous and modulated temperature rises in the sample.

The DC component of temperature decays as $1 / r$ around the heated particle, and the superimposed AC component $\Delta T$ has a similar behavior at low modulation frequencies. At higher frequencies however, in the "thermal waves" approach ${ }^{32}$, the modulated compo- 
nent of the temperature $\Delta T$ spreads over shorter lateral distances described by the thermal diffusion length $\mu=\sqrt{D / \pi F_{\text {heat }}}$, where $D$ is the thermal diffusivity. In e.g. glass $\left(D \approx 8 \times 10^{-7} \mathrm{~m}^{2} \cdot \mathrm{s}^{-1}\right), \Delta T$ is therefore confined within tens of micrometers around the structure above modulation frequencies $F_{\text {heat }}$ of the order of the $\mathrm{kHz}$. The modulated part of the scattered intensity, $\Delta I_{\text {scat }}$, therefore carries information on the local temperature of the nanostructure. At $1 \mathrm{kHz}$, the constant component of temperature is typically 1 order of magnitude lower than the modulated part of the temperature distribution for the structures studied in this work. Using the formalism proposed by Berto et al. ${ }^{38}$, we also verified that the contribution of neighboring nanostructures to the modulated temperature increase was low enough to be neglected, and could be compensated if necessary. Nevertheless, the $\mu$ m-range distance over which light interacts with this hot region can be much larger than the nm-range of the nano-objects, which ensures good signal-to-noise ratios $^{39}$.

Here, we propose to use a spatially resolved measurement of $\Delta I_{\text {scat }}$ in order to retrieve the local temperature variations $\Delta T$. Holography is able to image both the amplitude and the phase, and thus gives access to optical reconstructions of the scattered light modulation $\Delta I_{\text {scat }}$ in the plane of the scattering structure, or above it. However, most cameras are limited to frame rates of a few tens of Hertz, particularly in low-light conditions, and cannot directly measure kHz modulations. For this reason, we use a heterodyning scheme in which the probe illumination is modulated in order to create a low-frequency beating with the relevant modulation of $\Delta I_{\text {scat }}$, as detailed below (Figure 2).

\subsection{Experimental setup}

The experimental holographic setup is based on a Mach-Zehnder interferometer, schematically depicted in Figure 2 (c). The probe light source is a monomode laser diode at a wavelength $\lambda=785 \mathrm{~nm}$, (maximum power $P_{\max }=130 \mathrm{~mW}$ ) separated by a polarizing beam splitter (PBS) into reference and object arms polarized along the long axis of the nanostructures. The sample is illuminated through a prism in a total internal reflection configuration so that the illumination beam is reflected away and only the scattered light is collected by a microscope objective $(\mathrm{x} 100, \mathrm{NA}=0.8)$ which provides diffraction-limited spatial resolution at $785 \mathrm{~nm}$, typically over a 30x30 $\mu \mathrm{m}$ field of view. A beam splitter combines the scattered and reference light. The component of the scattered field which is parallel to the polarization of the reference interferes with the reference on the camera (SCMOS, Andor Zyla 5.5). A heterodyne digital holography configuration ${ }^{40-42}$ allows the implementation of a spatial ${ }^{43}$ and temporal filtering of the interference pattern $^{44}$. For this purpose, the reference and object arms are phase-modulated by Acousto-Optical Modulators (AOM1 and AOM2, respectively), at frequencies $f_{\mathrm{AOM} 1}$ and $f_{\mathrm{AOM} 2}$, both in the $80 \mathrm{MHz}$-range. If the sample is not heated, the interference pattern on the camera bears a modulation at the beating frequency $f_{\mathrm{AOM} 1}-f_{\mathrm{AOM} 2}$. Choosing closely spaced frequencies, e.g. $f_{\mathrm{AOM} 1}=80 \mathrm{MHz}$ and $f_{\mathrm{AOM} 2}=80 \mathrm{MHz}-3 \mathrm{~Hz}$ therefore induces a hologram beating at low frequency, $f_{\text {beat }}=3 \mathrm{~Hz}$. Using a $4 \times f_{\text {beat }}=12 \mathrm{~Hz}$ camera frame rate and 4-bucket acquisition, the slowly beating hologram can be demodulated and reconstructed in order to obtain images of the scattered optical field with an improved signal to noise ratio since low-frequency noises are filtered out. Heterodyne holography can provide shot-noise limited measurements ${ }^{40}$. In particular, when the imaging plane is at infinity, holography allows to reconstruct images while the information is spread over the whole imaging sensor. In the case of sparse scatterers, this allows to increase the illumination without saturating the few camera pixels imaging the object. When operating close to the saturation level of a pixel with a full-well capacity $\mathrm{N}=3 \times 10^{4}$ e-, 4-buckets demodulated images yield a SNR which varies as $\sqrt{4 N} \approx 350$ for each pixel and can be improved by spatial (binning) and/or temporal averaging.
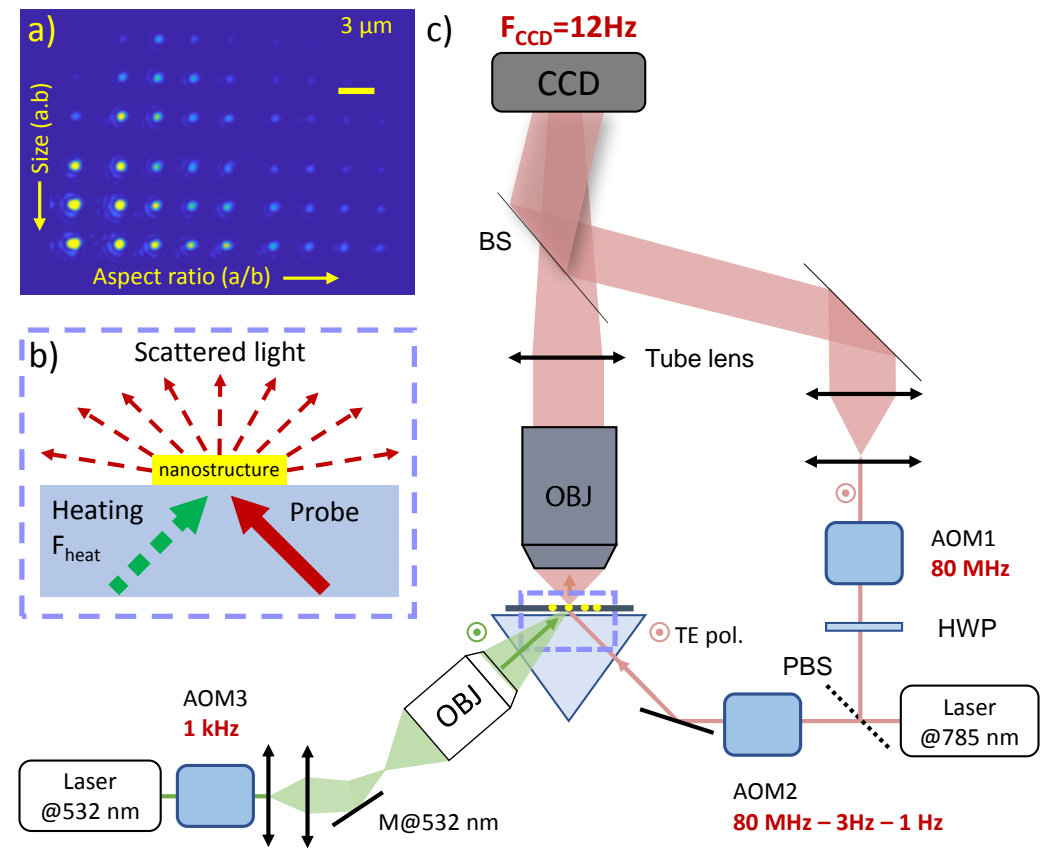

Figure 2. (a) Reconstructed hologram of an array of nanostructures, with increasing aspect ratio (left to right) and size (top to bottom), (b) Principle of heterodyne photothermal holography. (c) Schematic of the experimental setup. AOM: acousto-optic modulator; BS: beam 
splitter; M: mirror; OD: optical density; HWP: half-wave plate. The $532 \mathrm{~nm}$ modulated heating beam induces refractive index changes in the nanostructure, and therefore a modulation of the scattering of the probe beam $(785 \mathrm{~nm})$, from which temperature modulations are deduced. The red illumination and reference beams, as well as the green heating beam are polarized perpendicularly to the plane of the figure, and parallel to the long axis of the nanostructures.

Heterodyning also allows the measurement of high frequency components induced by heating with a powerful $\left(P_{\max }=2 \mathrm{~W}\right)$ singlemode laser at $\lambda=532 \mathrm{~nm}$, sinusoidally-modulated in amplitude by AOM3 at $F_{\text {heat }}=1 \mathrm{kHz}$. Accordingly, the frequency detuning of the reference and object arms $\Delta f=f_{\mathrm{AOM} 1}-f_{\mathrm{AOM} 2}$ has to be set to $\Delta f=f_{\text {beat }}+F_{\text {heat }}=1003 \mathrm{~Hz}$ in order to bring the $\Delta I_{\text {scat }}$ component which is originally modulated at $F_{\text {heat }}=1 \mathrm{kHz}$ down to a frequency of $f_{\text {beat }}=3 \mathrm{~Hz}$, which can be properly demodulated by the slow camera. Using this heterodyning technique, the components of the scattered optical field which are modulated at the heating frequency $F_{\text {heat }}=1 \mathrm{kHz}$ are retrieved. The same setup is therefore able to selectively retrieve holograms and images of the DC component of the scattered light $\mathrm{I}_{\mathrm{DCscat}}$ (for $\Delta f=3 \mathrm{~Hz}$ ) or of its AC component $I_{A C \text { scat }}$, which is thermally modulated $(\Delta f=1003$ $\mathrm{Hz}$ ) by simply modifying the detuning $\Delta f$.

\subsection{Signal processing and experimental temperature determination}

In the system considered in Figure 2 (b), the scattered field received at the detector can be written as:

$$
\mathbf{E}_{s}(\mathbf{r}, T)=k_{0}^{2} \int_{V} \mathbf{G}\left(\mathbf{r}, \mathbf{r}^{\prime}\right)\left[\epsilon\left(\mathbf{r}^{\prime}, T\right)-\epsilon_{m}\right] \mathbf{E}\left(\mathbf{r}^{\prime}, T\right) d^{3} r^{\prime}
$$

Here $\epsilon\left(\mathbf{r}^{\prime}, T\right)$ is the dielectric function of the nanorod, $\epsilon_{\mathrm{m}}$ is the dielectric function of the medium in which the nanorod is immersed, and $k_{0}=\omega / c$ with $\omega$ the optical frequency of the probe beam. The Green's function $\mathbf{G}\left(\mathbf{r}, \mathbf{r}^{\prime}\right)$ describes the optical response of the environment of the nanorod (including the substrate). The integral involves the total field $\boldsymbol{E}\left(\mathbf{r}^{\prime}, \mathrm{T}\right)$ and is extended over the volume $\mathrm{V}$ of the nanorod. The heating beam slightly changes $\epsilon\left(\mathbf{r}^{\prime}, \mathrm{T}\right)$ through its dependence on temperature, which in turns changes the amplitude of the scattered field. To first order, the change in the dielectric function can be written

$$
\epsilon\left(\mathbf{r}^{\prime}, T\right)=\epsilon\left(T_{0}\right)+\frac{\partial \epsilon}{\partial T}\left(T_{0}\right) \Delta T\left(\mathbf{r}^{\prime}\right)
$$

Where $T_{0}$ is the room temperature and $\Delta T=T-T_{0}$. Likewise, the change in the field inside the nanorod is:

$$
\mathbf{E}\left(\mathbf{r}^{\prime}, T\right)=\mathbf{E}\left(\mathbf{r}^{\prime}, T_{0}\right)+\frac{\partial E}{\partial T}\left(\mathbf{r}^{\prime}, T_{0}\right) \Delta T\left(\mathbf{r}^{\prime}\right)
$$

Due to the intensity modulation of the heating beam, $\Delta T$ is modulated at a frequency $F_{\text {heat }}$. The heterodyne holographic signal is proportional to the interference term $\operatorname{Re}\left[\Delta \mathbf{E}_{S}\left(\mathbf{r}, F_{\text {heat }}, T\right) . \mathbf{E}_{\text {ref }}^{*}\right]$ where $\mathbf{E}_{\text {ref }}$ is the reference field used to build the interferogram, and $\Delta \mathbf{E}_{s}\left(\mathbf{r}, F_{\text {heat }}, T\right)$ is the modulated part of the scattered field at the detector (here ${ }^{*}$ stands for the complex conjugate). The latter is obtained by inserting Eqs. (3) and (4) into Eq. (2):

$$
\begin{gathered}
\Delta \mathbf{E}_{s}\left(\mathbf{r}, F_{\text {heat }}, T\right)= \\
k_{0}^{2} \int_{V} \mathbf{G}\left(\mathbf{r}, \mathbf{r}^{\prime}\right) \frac{\partial \epsilon}{\partial \mathrm{T}}\left(T_{0}\right) \Delta T\left(\mathbf{r}^{\prime}\right) \mathbf{E}\left(\mathbf{r}^{\prime}, T_{0}\right) d^{3} r^{\prime}+k_{0}^{2} \int_{V} \mathbf{G}\left(\mathbf{r}, \mathbf{r}^{\prime}\right)\left[\epsilon\left(T_{0}\right)-\right. \\
\epsilon m] \partial \mathbf{E} \partial T\left(\mathbf{r}^{\prime}, T 0\right) \Delta T \mathbf{r}^{\prime} d 3 r^{\prime}
\end{gathered}
$$

Recovering $\Delta T$ from the signal requires the solution to a complex inverse problem. Here we follow a different approach, and use the simplest relevant model providing a direct estimate of $\Delta T$ from the data, in the particular case of small metallic nanorods.

For weakly scattering objects, the second term in the right-hand side of Eq. (5) can be neglected. To see this, we start by considering the Born approximation, that corresponds to single scattering inside the nanorod. This approximation amounts to replacing the field $\boldsymbol{E}\left(\mathbf{r}^{\prime}, T_{0}\right)$ in the integrals by the external field $\boldsymbol{E}_{0}\left(\mathbf{r}^{\prime}, T_{0}\right)$ in absence of the nanorod. The second term in the right-hand side of Eq. (5) vanishes in this simple case since $\boldsymbol{E}_{0}\left(\mathbf{r}^{\prime}, T_{0}\right)$ is not affected by external heating. The gold nanorods considered in this study cannot be treated in the Born approximation. Nevertheless, it can be seen that for optically small nanorods (with respect to the illumination wavelength), we can still expect the first integral in Eq. (5) to give the leading contribution to the signal. Consider the particular case of a sphere. In the quasi-static approximation, the internal field is homogeneous and well approximated by

$$
\mathbf{E}\left(\mathbf{r}_{0}, T\right) \approx \frac{3}{\epsilon(T)+2} \mathbf{E}_{0}\left(\mathbf{r}_{0}, T_{0}\right)
$$

where $\mathbf{r}_{0}$ is a point inside the sphere, so that

$$
\frac{\partial \mathbf{E}}{\partial T}\left(\mathbf{r}_{0}, T_{0}\right) \approx \frac{-3}{\left[\epsilon\left(T_{0}\right)+2\right]^{2}} \frac{\partial \epsilon}{\partial T}\left(T_{0}\right) \mathbf{E}_{0}\left(\mathbf{r}_{0}, T_{0}\right)
$$

Using the expressions above for the fields in the integrals in Eq. (5) shows that the second integral can be neglected provided that the condition $\left.\mid 3\left[\epsilon\left(T_{0}\right)-\epsilon_{m}\right] /\left[\epsilon\left(T_{0}\right)+2\right]^{2}\right] \mid \ll 1$ is fulfilled. Note that in practice, even a weak inequality in this condition is suffi- 
cient to provide a fair estimate, as justified by the good agreement between the measurements and full numerical simulations shown below. We conclude that for small nanorods satisfying this condition, we can write

$$
\Delta \mathbf{E}_{s}\left(\mathbf{r}, F_{\text {heat }}, T\right) \approx k_{0}^{2} \int_{V} \mathbf{G}\left(\mathbf{r}, \mathbf{r}^{\prime}\right) \frac{\partial \epsilon}{\partial \mathrm{T}}\left(T_{0}\right) \Delta T\left(\mathbf{r}^{\prime}\right) \mathbf{E}\left(\mathbf{r}^{\prime}, T_{0}\right) d^{3} r^{\prime}
$$

Inside a thermally small object, the temperature field is considered to be homogeneous. This occurs when the conduction time across the object $\tau_{c d}=L^{2} / \alpha$ (L being the size of the object and $\alpha$ is the thermal diffusivity) is smaller than the characteristic heating time $2 \pi / F_{\text {heat }}$, or in other words when $F_{\text {heat }} \ll 2 \pi \alpha / L^{2}$, which is satisfied in our experiment. In this case $\Delta T$ become independent on position. One can define the ratio of the modulated (AC) and the unmodulated (DC) signals as

$$
\eta=\frac{\operatorname{Re}\left[\Delta \mathbf{E}_{s}\left(\mathbf{r}, F_{\text {heat }}, T\right) \cdot \mathbf{E}_{\mathrm{ref}}^{*}\right]}{\operatorname{Re}\left[\Delta \mathbf{E}_{s}\left(\mathbf{r}, F=0, T_{0}\right) \cdot \mathbf{E}_{\mathrm{ref}}^{*}\right]}=\frac{\frac{\partial \epsilon}{\partial \mathrm{T}}\left(T_{0}\right) \Delta T}{\epsilon\left(T_{0}\right)-\epsilon_{m}} .
$$

From two measurements with and without modulated heating, we can therefore obtain the change in temperature $\Delta T$ inside the object as

$$
\Delta T=\eta \frac{\epsilon\left(T_{0}\right)-\epsilon_{m}}{\frac{\partial \epsilon}{\partial T}\left(T_{0}\right)} .
$$

\section{RESULTS}

\subsection{Numerical methods}

In order to model and validate the experimental results, two types of numerical optical simulations were used and compared: the Boundary Element Model (BEM) method $^{37}$, using the freely available Matlab-based package MNPBEM, and the Finite Difference Time Domain (FDTD) method, using the commercially available solver Lumerical solutions Inc. ${ }^{45}$ For each aspect ratio, the structures under study are considered as thermally and optically independent from neighboring objects, with substrate and structure materials, dimensions and illumination matching the performed experiments. For FDTD, the area surrounding the structures is discretized by a non-uniform conformal variant mesh with a meshing size of $1 \mathrm{~nm}$. The incident laser beams are modeled as plane waves. The simulation area was terminated by Perfectly Matched Layers (PMLs) to suppress spurious reflection from boundaries.

\subsection{Scattering cross-section}

The measurement of the $I_{D C \text { scat }}$ hologram without heating the sample reveals the scattering efficiency of the structures at the wavelength $\lambda=785 \mathrm{~nm}$. In order to obtain quantitative cross-section values, we used the average of measurements carried out on $N=4$ identical structures as reference, and used the dispersion of these results to evaluate the uncertainty. The 3D scattering pattern of the same structure was then modelled using FDTD method, taking into account the objective's collection angle for a numerical aperture of 0.8. Taking this calculated value and the average measurement for references, measurements for all aspect ratios were normalized to obtain quantitative scattering cross-section values. Subsequent measurements were carried out on individual sets of nanostructures using the same illumination power, collection N.A., and therefore the same normalization factor. Possible variations in collection efficiency due to changes in the shape of the scattering pattern for $A R \neq 1$ were not considered, and therefore contribute to possible discrepancies between the model and the experiment. Figure 3 shows the experimental results obtained for the structures of varying aspect ratio described in Figure 1 along with the corresponding numerical simulations. As expected, the scattering crosssections display a decrease with the aspect ratio, as the resonance shifts away from the probe wavelength $(785 \mathrm{~nm})$ into the infrared. 


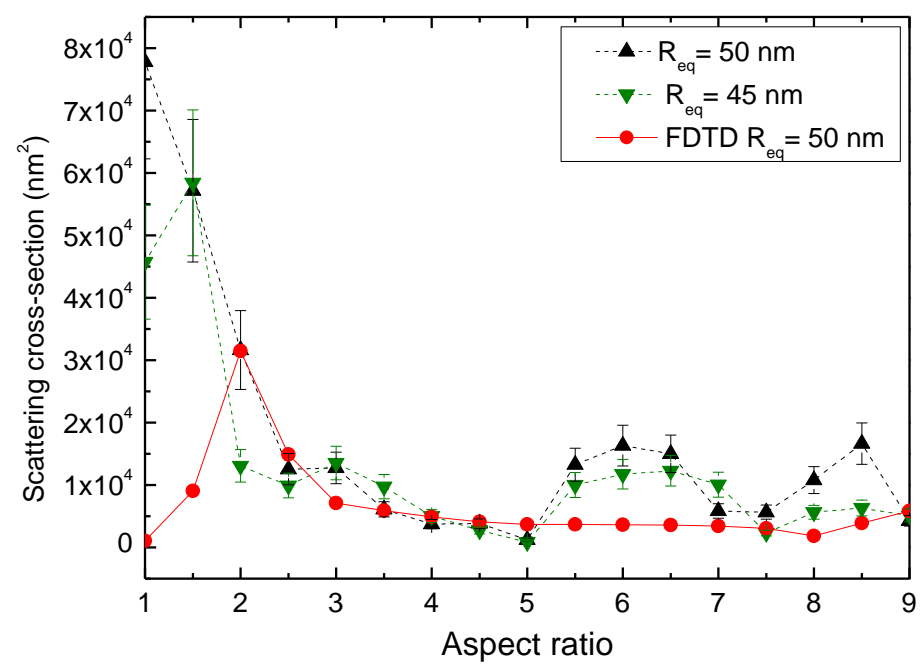

Figure 3. Experimental (dotted line) and modelled (continuous line) scattering cross sections as a function of nanostructure aspect ratio for a polarization along the long axis of the nanostructures. $\mathrm{R}_{\mathrm{eq}}$ denotes the targeted radius of the $\mathrm{AR}=1$ disc. Experimental measurements are given for $\mathrm{R}_{\mathrm{eq}}=45$ and $50 \mathrm{~nm}$ to illustrate deviations induced by the $5 \mathrm{~nm}$ fabrication precision.

\subsection{Temperature measurements}

As described above, the modulated component of the temperature increase can be evaluated using heterodyne measurements of $\mathrm{I}_{\mathrm{ACscat}}$ to derive the values of the dimensionless ratio $\eta$ defined in Eq. (9), a process which does not require the use of the model for calibration.

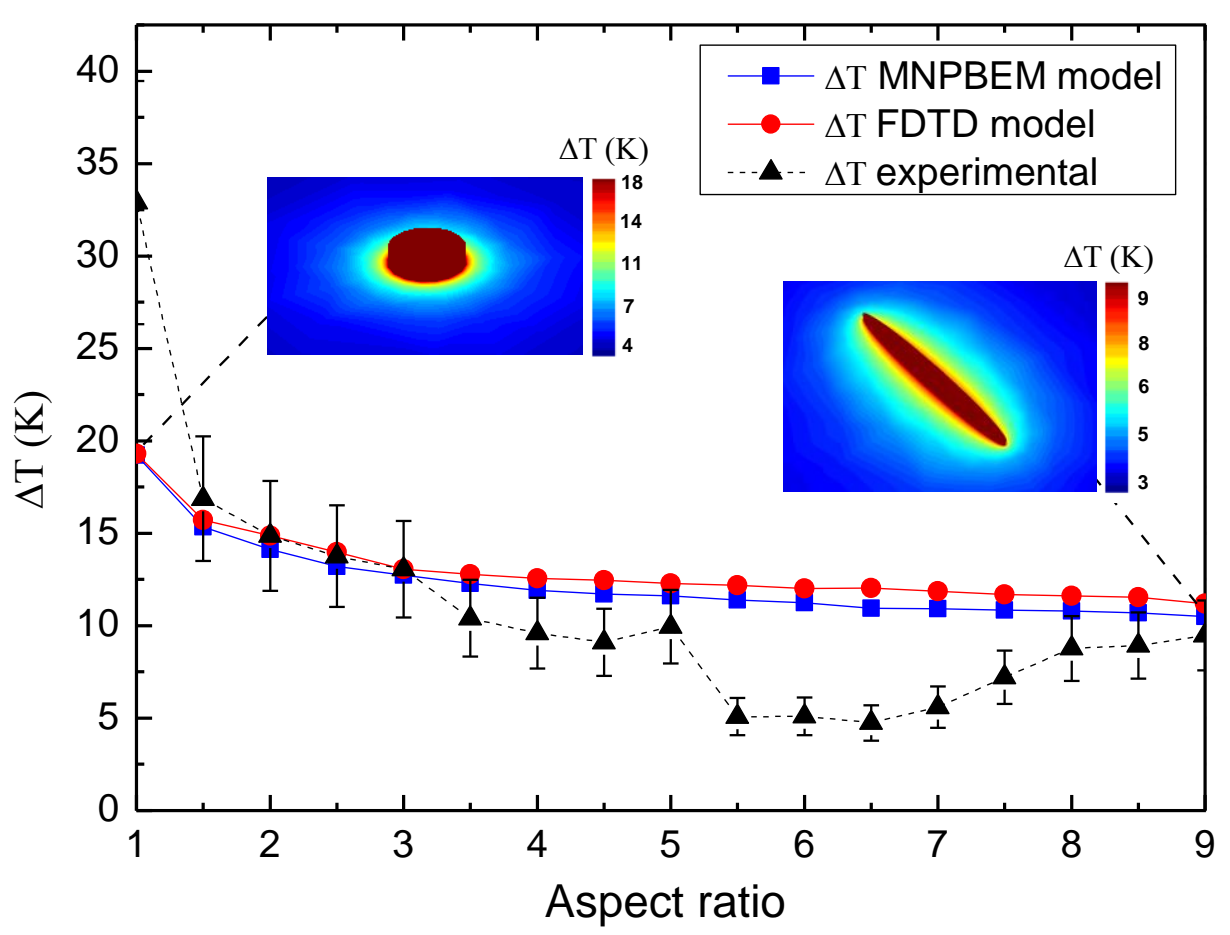

Figure 4. Experimental temperature variations along with the corresponding error, compared to FDTD and BEM -based modelling, for an irradiance $I_{\text {heat }}=2.36 \times 10^{8} \mathrm{~W} / \mathrm{m}^{2}$. Note that no parameter is adjusted in the simulations. Experimental error bars correspond to the standard deviation over measurements carried out on 4 independent samples. 
The value of $\frac{\partial \varepsilon}{\partial T}$ for gold at the probe wavelength $(785 \mathrm{~nm})$ was deduced from experimental data ${ }^{46}, \frac{d n}{d T}=1.2 \times 10^{-4} \mathrm{~K}^{-1}$ at $\lambda=785 \mathrm{~nm}$. Note that for borosilicate glasses, $\frac{d n}{d T}$ is relatively constant with wavelength and is 2 orders of magnitude lower in the visible at $3 \times 10^{-6} \mathrm{~K}^{-1}$, and can therefore be neglected ${ }^{47}$. Varying irradiance conditions caused by the position of nanostructures with respect to the Gaussian heating beam were taken into account and corrected to allow comparisons at equivalent irradiance $I_{\text {heat }}=2.36 \times 10^{8}$ $\mathrm{W} / \mathrm{m}^{2}$, for a polarization of the heating beam parallel to the long axis of the nanorods.Figure 4 shows the experimental temperature measurements obtained in gold nanorods, determined using Eq. (10), as a function of the structure aspect ratio. For each of the 17 aspect ratios and for 2 equivalent radii $(45$ and $50 \mathrm{~nm}$ ), 4 different structures were fabricated and characterized. A standard deviation analysis on these $17 \times 2 \times 4=136$ measurements yields uncertainties of $20 \%$ as plotted in Figures 3 and 4 .

The measurements shown here were obtained using temporal averaging over $\mathrm{n}=100$ series of 4 images, i.e. $\Delta \mathrm{t}=33 \mathrm{~s}$ at $f_{\text {beat }}=$ $12 \mathrm{~Hz}$, which improves SNR by a factor $\sqrt{n}=10$ as compared to a single 4 -image series ( $\Delta \mathrm{t}=333 \mathrm{~ms}$ ). Experimentally, we measured the repeatability of the measurement on 9 different structures. We obtained a noise $\delta T(\Delta \mathrm{t})$ which varies in good agreement with a $1 / \sqrt{\Delta t}$ (or $1 / \sqrt{n}$ ) law, with $\delta T(0.333 \mathrm{~s})=1.6 \mathrm{~K}, \delta T(6.7 \mathrm{~s})=0.6, \delta T(16.7 \mathrm{~s})=0.35 \mathrm{~K}$, and $\delta T(33 \mathrm{~s})=0.3 \mathrm{~K}$, corresponding to $\mathrm{n}$ $=1,20,50$ and 100 respectively. This clearly indicates that the temperature fluctuations observed in Figure 4 are not caused by measurement uncertainty, but merely by deviations from the targeted geometry during the fabrication, which is consistent with both SEM and spectroscopic measurements.

To compare the performances of optical methods potentially limited by photon noise (table 1 in reference ${ }^{48}$ ), we therefore propose to consider $\delta T \cdot \sqrt{\Delta t}$ as a criterion to be minimized. With $\delta T . \sqrt{\Delta t} \approx 1.3 \mathrm{~K} \cdot \mathrm{s}^{1 / 2}$, our method performs similarly to liquid crystals and TRSTM $\left(1 K . s^{1 / 2}\right)$, which are physically and thermally invasive methods, and notably better than Raman spectroscopy (100 K.s ${ }^{1 / 2}$ ). Finally, keeping in mind that holography measures typ. $N_{\text {pix }}=1024^{2}$ pixels at once, a $\delta T \cdot \sqrt{\Delta t} / N_{p i x}$ criterion arguably gives a clear advantage to such full-field methods in terms of temperature sensitivity per point and unit of time.

In order to quantify accurately temperatures at the nanoscale and verify the experimental approach, the results are compared to two numerical simulations. In these two simulations, radiative transfer and ballistic transport are considered to be negligible compared to heat diffusion. In the first approach, we calculated the absorption cross section, $\sigma_{\mathrm{abs}}$, using the FDTD method. This cross section was then used to calculate the heat source term $P$, i.e. the absorbed power $P=\sigma_{\text {abs }} I_{\text {heat }}$, with $I_{\text {heat }}=2.36 \times 10^{8} \mathrm{~W} / \mathrm{m}^{2}$ the irradiance used in the experiments. The 3D temperature map was then calculated using the Heat Transport solver (HEAT) from Lumerical, ${ }^{45}$ which solves the heat diffusion equation using a Finite Elements Method and a meshing of the system. The temperature at the bottom of the substrate was set at $300 \mathrm{~K}$, and the effective boundary conditions between solids and air were set as convective. By varying the heating frequency down to values in the $\mathrm{Hz}$ range, we verified that steady-state heating and high frequency modulation of the heating $(1 \mathrm{kHz})$ yield very similar temperatures, which is expected since the involved spatial scales (nm-range) are well below the thermal diffusion lengths, even at $F_{\text {heat }}=1 \mathrm{kHz}$. The inset in Figure (3) shows the 3D temperature map obtained for two nanostructures with aspect ratios 1 and 9. It confirms the validity of the uniform temperature approximation within the particle, which was used to determine the temperature experimentally and in the following MNPBEM numerical approach. This second approach is based on calculations performed with the freely available MNPBEM14 software package, ${ }^{37}$ in which the absorption cross section $\sigma_{\mathrm{abs}}$ is determined by a boundary element method, then under the uniform temperature approximation, the temperature elevation in the structures can be calculated using

$$
\Delta T=\frac{\sigma_{a b s} I_{\text {heat }}}{4 \pi \bar{\kappa} R_{L}}
$$

With $\bar{\kappa}=\frac{\kappa_{1}+\kappa 2}{2}$, the effective thermal conductivity of the medium ( $\kappa_{1}$ and $\kappa_{2}$ being, respectively, the thermal conductivities of the substrate and air), and $R_{L}$ the Laplace radius of the numerically meshed nanostructure. ${ }^{49}$ Here, one can define the so-called Laplace matrix as $\boldsymbol{A}_{i j}=\frac{1}{\left|\boldsymbol{r}_{i}-\boldsymbol{r}_{j}\right|}$ for $i \neq j$, and $\frac{2}{a}$ for $i=j$, where, $\boldsymbol{r}_{i}$ is the position vector of the mesh element $i$ and $a$ is the grid constant. Then $R_{L}$ is obtained by summing the elements of the inverted matrix:

$$
R_{L}=\sum_{i, j}^{N}\left(\boldsymbol{A}^{-1}\right)_{i j} \text {. }
$$

\section{CONCLUSION}

The comparison of experimental and calculated temperatures is generally satisfactory, considering the fact that individual nanostructures are investigated. However, some discrepancies appear for aspect ratios of 6 and above. Despite the high precision of electron beam lithography, some deviations from the required dimensions are unavoidable, and were quantified using SEM images at typically $\pm 5 \mathrm{~nm}$. This is most significant for the thinnest and elongated structures in which metal continuity can be interrupted, and where the targeted elliptic shape is distorted. Another source of error is the approximation made in Eq. (8), where the second integral term in Eq. (5) induced by the modulation of the internal field is neglected. Whenever the condition $\mid 3\left[\epsilon\left(T_{0}\right)-\epsilon_{m}\right] /$ $\epsilon T 0+22] \ll 1$ is fulfilled, however, this approximation provides a simple way to derive quantitative temperatures which are in remarkably good agreement with experiments considering that no adjustable parameter is used. 
Using a far-field optical microscopy technique based on heterodyne detection of the modulated scattered electric field, we have proposed a methodology to determine quantitatively the temperature variations of single nanostructures under modulated optical excitation. Since the method is holographic, it also gives access to the optical path difference, although this information was not exploited here. In the DC regime, phase information has already proven its ability to yield sensitive temperature measurements ${ }^{29}$ : its exploitation is a promising way to further improve sensitivity in modulated regimes.

Funding sources

French Agence Nationale de la Recherche (ANR NATO, Grant ANR-13-BS10-0013-03).

\section{ACKNOWLEDGMENT}

The fabrication and electron microscopy were performed at nano'mat - UTT, Serguei Kochtcheev and Jérémie Béal are acknowledged for their assistance. We thank Guillaume Baffou for his inputs at early stages of this work and for his critical reading of the manuscript.

\section{REFERENCES}

(1) Lal, S.; Clare, S. E.; Halas, N. J. Nanoshell-Enabled Photothermal Cancer Therapy: Impending Clinical Impact. Acc. Chem. Res. 2008, 41 (12), 1842-1851. https://doi.org/10.1021/ar800150g.

(2) Cherukuri, P.; Glazer, E. S.; Curley, S. A. Targeted Hyperthermia Using Metal Nanoparticles. Adv. Drug Deliv. Rev. 2010, 62 (3), 339-345. https://doi.org/10.1016/j.addr.2009.11.006.

(3) Huang, X.; Jain, P. K.; El-Sayed, I. H.; El-Sayed, M. A. Plasmonic Photothermal Therapy (PPTT) Using Gold Nanoparticles. Lasers Med. Sci. 2008, 23 (3), 217-228. https://doi.org/10.1007/s10103-007-0470-x.

(4) Urban, A. S.; Pfeiffer, T.; Fedoruk, M.; Lutich, A. A.; Feldmann, J. Single-Step Injection of Gold Nanoparticles through Phospholipid Membranes. ACS Nano 2011, 5 (5), 3585-3590. https://doi.org/10.1021/nn201132a.

(5) Zograf, G. P.; Timin, A. S.; Muslimov, A. R.; Shishkin, I. I.; Nominé, A.; Ghanbaja, J.; Ghosh, P.; Li, Q.; Zyuzin, M. V.; Makarov, S. V. All-Optical Nanoscale Heating and Thermometry with Resonant Dielectric Nanoparticles for Controllable Drug Release in Living Cells. Laser Photonics Rev. 2020, 14 (3), 1900082. https://doi.org/10.1002/lpor.201900082.

(6) Timko, B. P.; Dvir, T.; Kohane, D. S. Remotely Triggerable Drug Delivery Systems. Adv. Mater. 2010, 22 (44), 4925-4943. https://doi.org/10.1002/adma.201002072.

(7) Han, G.; Ghosh, P.; De, M.; Rotello, V. M. Drug and Gene Delivery Using Gold Nanoparticles. NanoBiotechnology 2007, 3 (1), 40-45. https://doi.org/10.1007/s12030-007-0005-3.

(8) Cognet, L.; Berciaud, S.; Lasne, D.; Lounis, B. Photothermal Methods for Single Nonluminescent Nano-Objects. Anal. Chem. 2008, 80 (7), 2288-2294. https://doi.org/10.1021/ac086020h.

(9) COPLAND, J.; EGHTEDARI, M.; POPOV, V.; KOTOV, N.; MAMEDOVA, N.; MOTAMEDI, M.; ORAEVSKY, A. Bioconjugated Gold Nanoparticles as a Molecular Based Contrast Agent: Implications for Imaging of Deep Tumors Using Optoacoustic Tomography. Mol. Imaging Biol. 2004, 6 (5), 341-349. https://doi.org/10.1016/j.mibio.2004.06.002.

(10) Challener, W. A.; Peng, C.; Itagi, A. V.; Karns, D.; Peng, W.; Peng, Y.; Yang, X.; Zhu, X.; Gokemeijer, N. J.; Hsia, Y. T.; Ju, G.; Rottmayer, R. E.; Seigler, M. A.; Gage, E. C. Heat-Assisted Magnetic Recording by a near-Field Transducer with Efficient Optical Energy Transfer. Nat. Photonics 2009, 3 (4), 220-224. https://doi.org/10.1038/nphoton.2009.26.

(11) Cao, L.; Barsic, D. N.; Guichard, A. R.; Brongersma, M. L. Plasmon-Assisted Local Temperature Control to Pattern Individual Semiconductor Nanowires and Carbon Nanotubes. Nano Lett. 2007, 7 (11), 3523-3527. https://doi.org/10.1021/nl0722370.

(12) Wang, L.; Li, B. Thermal Memory: A Storage of Phononic Information. Phys. Rev. Lett. 2008, 101 (26), 267203. https://doi.org/10.1103/PhysRevLett.101.267203.

(13) Donner, J. S.; Baffou, G.; McCloskey, D.; Quidant, R. Plasmon-Assisted Optofluidics. ACS Nano 2011, 5 (7), $5457-5462$. https://doi.org/10.1021/nn200590u.

(14) Liu, C.; Tessier, G.; Flores Esparza, S. I.; Guillon, M.; Berto, P. Reconfigurable Temperature Control at the Microscale by Light Shaping. ACS Photonics 2019, 6 (2), 422-428. https://doi.org/10.1021/acsphotonics.8b01354.

(15) Williams, C. C.; Wickramasinghe, H. K. Scanning Thermal Profiler. Microelectron. Eng. 1986, 5 (1-4), 509-513. https://doi.org/10.1016/0167-9317(86)90084-5.

(16) Baffou, G.; Girard, C.; Quidant, R. Mapping Heat Origin in Plasmonic Structures. Phys. Rev. Lett. 2010, 104 (13), 136805. 
https://doi.org/10.1103/PhysRevLett.104.136805.

(17) Carlson, M. T.; Khan, A.; Richardson, H. H. Local Temperature Determination of Optically Excited Nanoparticles and Nanodots. Nano Lett. 2011, 11 (3), 1061-1069. https://doi.org/10.1021/nl103938u.

(18) Ross, D.; Gaitan, M.; Locascio, L. E. Temperature Measurement in Microfluidic Systems Using a Temperature-Dependent Fluorescent Dye. Anal. Chem. 2001, 73 (17), 4117-4123. https://doi.org/10.1021/ac010370l.

(19) Brites, C. D. S.; Lima, P. P.; Silva, N. J. O.; Millán, A.; Amaral, V. S.; Palacio, F.; Carlos, L. D. Thermometry at the Nanoscale. Nanoscale. 2012, pp 4799-4829. https://doi.org/10.1039/c2nr30663h.

(20) Vetrone, F.; Naccache, R.; Zamarrón, A.; De La Fuente, A. J.; Sanz-Rodríguez, F.; Maestro, L. M.; Rodriguez, E. M.; Jaque, D.; Sole, J. G.; Capobianco, J. A. Temperature Sensing Using Fluorescent Nanothermometers. ACS Nano 2010, 4 (6), 3254-3258. https://doi.org/10.1021/nn100244a.

(21) Jaque, D.; Vetrone, F. Luminescence Nanothermometry. $\quad$ Nanoscale $\quad 2012$, $4 \quad$ (15), 4301. https://doi.org/10.1039/c2nr30764b.

(22) Zograf, G. P.; Petrov, M. I.; Zuev, D. A.; Dmitriev, P. A.; Milichko, V. A.; Makarov, S. V.; Belov, P. A. Resonant Nonplasmonic Nanoparticles for Efficient Temperature-Feedback Optical Heating. Nano Lett. 2017, 17 (5), $2945-2952$. https://doi.org/10.1021/acs.nanolett.7b00183.

(23) Carattino, A.; Caldarola, M.; Orrit, M. Gold Nanoparticles as Absolute Nanothermometers. Nano Lett. 2018, 18 (2), 874-880. https://doi.org/10.1021/acs.nanolett.7b04145.

(24) Hugall, J. T.; Baumberg, J. J. Demonstrating Photoluminescence from $\mathrm{Au}$ Is Electronic Inelastic Light Scattering of a Plasmonic Metal: The Origin of SERS Backgrounds. Nano Letters. 2015, pp 2600-2604. https://doi.org/10.1021/acs.nanolett.5b00146.

(25) Xie, X.; Cahill, D. G. Thermometry of Plasmonic Nanostructures by Anti-Stokes Electronic Raman Scattering. Appl. Phys. Lett. 2016, 109 (18), 183104. https://doi.org/10.1063/1.4966289.

(26) Pozzi, E. A.; Zrimsek, A. B.; Lethiec, C. M.; Schatz, G. C.; Hersam, M. C.; Van Duyne, R. P. Evaluating Single-Molecule Stokes and Anti-Stokes SERS for Nanoscale Thermometry. J. Phys. Chem. C 2015, 119 (36), 21116-21124. https://doi.org/10.1021/acs.jpcc.5b08054.

(27) Milichko, V. A.; Zuev, D. A.; Baranov, D. G.; Zograf, G. P.; Volodina, K.; Krasilin, A. A.; Mukhin, I. S.; Dmitriev, P. A.; Vinogradov, V. V.; Makarov, S. V.; Belov, P. A. Metal-Dielectric Nanocavity for Real-Time Tracing Molecular Events with Temperature Feedback. Laser Photonics Rev. 2018, 12 (1), 1700227. https://doi.org/10.1002/lpor.201700227.

(28) Boyer, D. Photothermal Imaging of Nanometer-Sized Metal Particles Among Scatterers. Science (80-. ). 2002, 297 (5584), 1160-1163. https://doi.org/10.1126/science.1073765.

(29) Baffou, G.; Bon, P.; Savatier, J.; Polleux, J.; Zhu, M.; Merlin, M.; Rigneault, H.; Monneret, S. Thermal Imaging of Nanostructures by Quantitative Optical Phase Analysis. ACS Nano 2012, 6 (3), 2452-2458. https://doi.org/10.1021/nn2047586.

(30) Gaiduk, A.; Yorulmaz, M.; Ruijgrok, P. V.; Orrit, M. Room-Temperature Detection of a Single Molecule's Absorption by Photothermal Contrast. Science (80-. ). 2010, 330 (6002), 353-356. https://doi.org/10.1126/science.1195475.

(31) Berto, P.; Ureña, E. B.; Bon, P.; Quidant, R.; Rigneault, H.; Baffou, G. Quantitative Absorption Spectroscopy of Nano-Objects. Phys. Rev. B 2012, 86 (16), 165417. https://doi.org/10.1103/PhysRevB.86.165417.

(32) Rosencwaig, A.; Gersho, A. Theory of the Photoacoustic Effect with Solids. J. Appl. Phys. 1976, 47 (1), 64-69. https://doi.org/10.1063/1.322296.

(33) Tessier, G. Photothermal Techniques. In Topics in Applied Physics; 2009; pp 389-409. https://doi.org/10.1007/978-3-64204258-4_13.

(34) Lalisse, A.; Tessier, G.; Plain, J.; Baffou, G. Quantifying the Efficiency of Plasmonic Materials for Near-Field Enhancement and Photothermal Conversion. J. Phys. Chem. C 2015, 119 (45), 25518-25528. https://doi.org/10.1021/acs.jpcc.5b09294.

(35) Brasiliense, V.; Berto, P.; Combellas, C.; Tessier, G.; Kanoufi, F. Electrochemistry of Single Nanodomains Revealed by ThreeDimensional Holographic Microscopy. Acc. Chem. Res. 2016, 49 (9), 2049-2057. https://doi.org/10.1021/acs.accounts.6b00335. 
(36) Brasiliense, V.; Berto, P.; Combellas, C.; Kuszelewicz, R.; Tessier, G.; Kanoufi, F. Electrochemical Transformation of Individual Nanoparticles Revealed by Coupling Microscopy and Spectroscopy. Faraday Discuss. 2016, 193, 339-352. https://doi.org/10.1039/c6fd00098c.

(37) García de Abajo, F. J.; Howie, A. Retarded Field Calculation of Electron Energy Loss in Inhomogeneous Dielectrics. Phys. Rev. B 2002, 65 (11), 115418. https://doi.org/10.1103/PhysRevB.65.115418.

(38) Berto, P.; Mohamed, M. S. A.; Rigneault, H.; Baffou, G. Time-Harmonic Optical Heating of Plasmonic Nanoparticles. Phys. Rev. B 2014, 90 (3), 035439. https://doi.org/10.1103/PhysRevB.90.035439.

(39) Berciaud, S.; Lasne, D.; Blab, G. A.; Cognet, L.; Lounis, B. Photothermal Heterodyne Imaging of Individual Metallic Nanoparticles: Theory versus Experiment. Phys. Rev. B 2006, 73 (4), 045424 . https://doi.org/10.1103/PhysRevB.73.045424.

(40) Gross, M.; Atlan, M. Digital Holography with Ultimate Sensitivity. Opt. Lett. 2007, 32 (8), 909. https://doi.org/10.1364/OL.32.000909.

(41) Lalisse, A. Optimisation Thermique de Nanostructures Plasmoniques: Conception, Modélisation et Caractérisation, Université Pierre et Marie Curie, 2017.

(42) Suck, S. Y. Digital Heterodyne Holography for Plasmonic Nanostructures, Université Pierre et Marie Curie, 2011.

(43) Cuche, E.; Marquet, P.; Depeursinge, C. Spatial Filtering for Zero-Order and Twin-Image Elimination in Digital off-Axis Holography. Appl. Opt. 2000, 39 (23), 4070. https://doi.org/10.1364/ao.39.004070.

(44) Suck, S. Y.; Collin, S.; Bardou, N.; Wilde, Y. De; Tessier, G. Imaging the Three-Dimensional Scattering Pattern of Plasmonic Nanodisk Chains by Digital Heterodyne Holography. Opt. Lett. 2011, 36 (6), 849. https://doi.org/10.1364/OL.36.000849.

(45) Lumerical Inc. http://www.lumerical.com.

(46) Johnson, P.; Christy, R. Optical Constants of Transition Metals: Ti, V, Cr, Mn, Fe, Co, Ni, and Pd. Phys. Rev. B 1974, 9 (12), 5056-5070. https://doi.org/10.1103/PhysRevB.9.5056.

(47) Jewell, J. M. Thermooptic Coefficients of Some Standard Reference Material Glasses. J. Am. Ceram. Soc. 1991, 74 (7), 16891691. https://doi.org/10.1111/j.1151-2916.1991.tb07162.x.

(48) Van De Broek, B.; Grandjean, D.; Trekker, J.; Ye, J.; Verstreken, K.; Maes, G.; Borghs, G.; Nikitenko, S.; Lagae, L.; Bartic, C.; Temst, K.; Van Bael, M. J. Temperature Determination of Resonantly Excited Plasmonic Branched Gold Nanoparticles by XRay Absorption Spectroscopy. Small 2011, 7 (17), 2498-2506. https://doi.org/10.1002/smll.201100089.

(49) Baffou, G.; Quidant, R.; Girard, C. Thermoplasmonics Modeling: A Green's Function Approach. Phys. Rev. B 2010, 82 (16), 165424. https://doi.org/10.1103/PhysRevB.82.165424. 
TOC Graphic

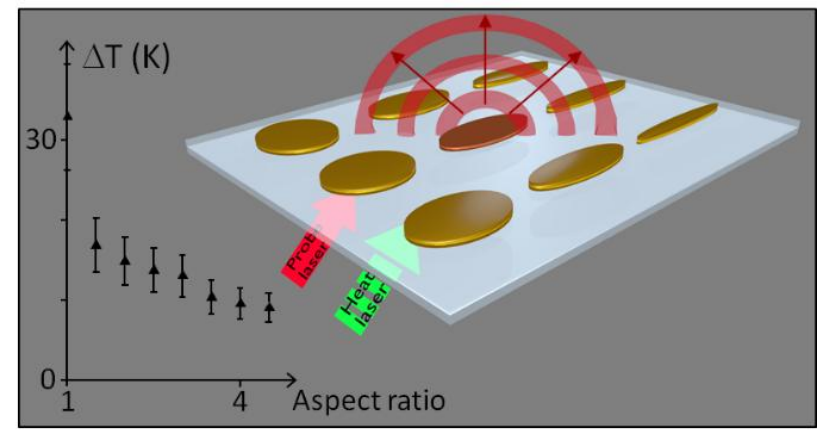

